\title{
Meiotic Instability in the Microsporocytes of an Aberrant Plant Isolated from the Mutant Progeny of Nigella sativa L.
}

\author{
Animesh K. Datta and Amal K. Biswas \\ Department of Botany, University of Kalyani, Kalyani-741235, India
}

Accepted March 27, 1984

Present authors were investigating the cytogenetical aspects of mutation breeding in Nigella sativa L. (black cummin), a widely grown spice yielding plant in the family Ranunculaceae, and have been able to recover some mutant lines in the species. One of them was a EMS -induced (ethyl methane sulphonate) lax branching mutant showing cytomixis during prophase $I$ and formation of various hypo- and hyperploid PMCs at the subsequent stages (Datta and Biswas 1984). The $\mathbf{M}_{3}$ segregating progeny raised from the selfed seeds of the mutant produced two phenotypically aberrant looking plants $(0.65 \%)$; one of which was cytologically confirmed as a trisomic (Datta and Biswas 1984) and the anomalous cytomorphological behaviour of the other plant is described in the present communication.

\section{Materials and methods}

A phenotypically aberrant plant has been isolated from the selfed progeny of the $\mathrm{M}_{2}$ lax branching (EMS-induced) mutant of Nigella sativa $\mathrm{L}$. Morphological nature of the phenotypic marker plant was noted. Studies on the pollen and pollen mother cells were made following the same procedure as was followed by the authors earlier (1983).

Due to paucity of anthers and clumping of chromosomes, sufficient number of meiocytes in the aberrant plant could not be analyzed at metaphase I and none of them was recovered from the subsequent stages. Similar types of variations were noted from different anthers when single microsporophyll was squashed per slide and the result has been formulated from the total count of all the PMCs excluding those with clumped and sticky appearance of chromosomes.

\section{Results and discussion}

Phenotypically, the aberrant plant exhibited lax branching nature attaining a relatively shorter height $(32.7 \mathrm{~cm})$ at maturity as compared to rather erect $(43.65 \mathrm{~cm}$ \pm 1.72 ) and compact habit of the normal plants (Figs. 1 and 2). During the initial growth period of the plant the pinnae of lamina were represented by linear, thicker, appendage-like structures and at the later stages, few normal leaves developed (Fig. 2). Most of the flower buds terminated in rudimentary flowers excepting a few which bloomed after 121-137 days after sowing instead of 70-98 days in control 
plants. The flowers had only 1-2 normal looking stamens, while rest of the microsporophylls were represented as leafy projections. These flowers produced only rudimentary capsules with abortive seeds.
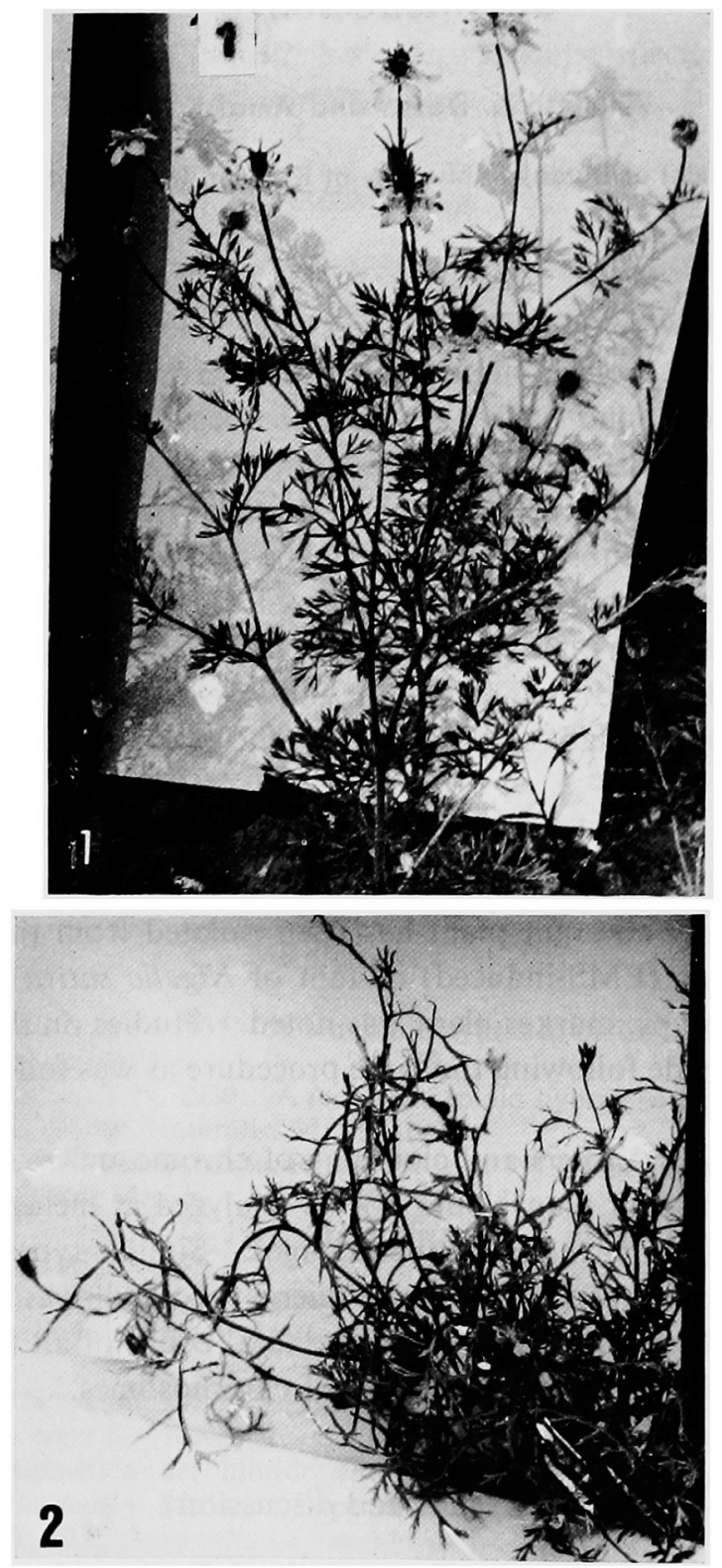

Figs. 1-2. Mature plants of Nigella sativa L. 1, control plant. 2, phenotypically marked aberrant plant showing lax tranching nature and leaf deformity.

The control plants revealed mostly 6 bivalents at metaphase I (Fig. 3) and rare occurrence of 2 or 4 univalents possibly due to precocious separations of chromosomes. Clumping and stickiness of chromosomes occurred predominantly in the meiotic cells of the phenotypic marker plant (Figs. 4 and 5); however, meiotic analy- 


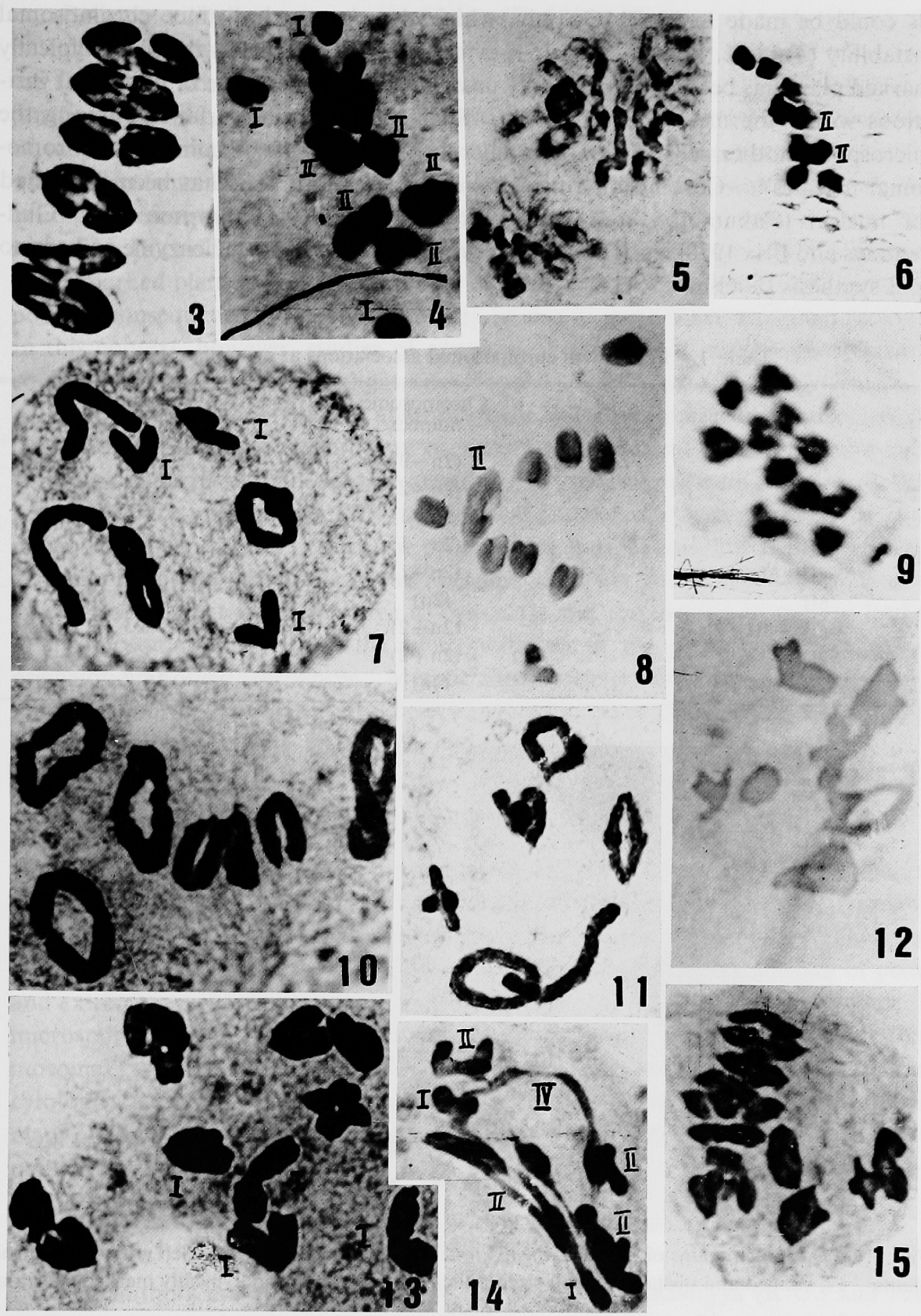

Figs. 3-15. Metaphase I configurations in N. sativa. 3, 6II in control plants. 4-15. Male meiosis in the marker plant. 4, PMC showing clumping tendency with more than 12 chromosomes appearing as univalents and bivalents. 5, sticky behaviour of chromosomes showing variations in chromosome number in two adjacent meiocytes. 6, 2II +5I. 7, 4II +3I. 8, 1II +9I. 9, 11I. 10 and $11,6 \mathrm{II}$. 12, 7II. 13, 4II +4I. 14, 1IV +4II +1I. 15, $12 \mathrm{II}$. 
sis could be made in 78 PMCs only, which demonstrated distinct chromosomal instability (Table 1, Figs. 6-15). It is evident from Table 1 that the phenotypically marked plant has become cytologically unstable due to prevalence of numerical variations within the meiotic complement. Such chromosomal variations among the microspore mother cells of the same microsporophyll may be coined as "chromosomal mosaics". Chromosome mosaicism in the meiotic cells has been attributed for nuclear (Fukumoto 1962) and genotypic instability (Thompson 1962, Guildenhuys and Brix 1958); or it might be resulted due to unbalanced enzyme and amino acid synthesis (Nielsen 1961).

Table 1. PMCs with chromosomal associations at metaphase I

\begin{tabular}{|c|c|c|}
\hline Configurations & $\begin{array}{c}\text { Chromosome } \\
\text { number }\end{array}$ & $\begin{array}{c}\text { Frequency } \\
(\%)\end{array}$ \\
\hline $2 \mathrm{II}+5 \mathrm{I}$ & $(2 n-3)$ & 2.6 \\
\hline 5II & $(2 n-2)$ & 5.3 \\
\hline $11 \mathrm{I}$ & $(2 n-1)$ & 2.6 \\
\hline $5 \mathrm{II}+1 \mathrm{I}$ & $(2 n-1)$ & 7.9 \\
\hline $4 \mathrm{II}+3 \mathrm{I}$ & $(2 n-1)$ & 7.9 \\
\hline $6 \mathrm{II}$ & $(2 n)$ & 26.3 \\
\hline $1 I V+4 I I+2 I$ & $(2 n+2)$ & 5.3 \\
\hline 7 II & $(2 n+2)$ & 5.3 \\
\hline $12 \mathrm{II}$ & $(4 n)$ & 36.8 \\
\hline
\end{tabular}

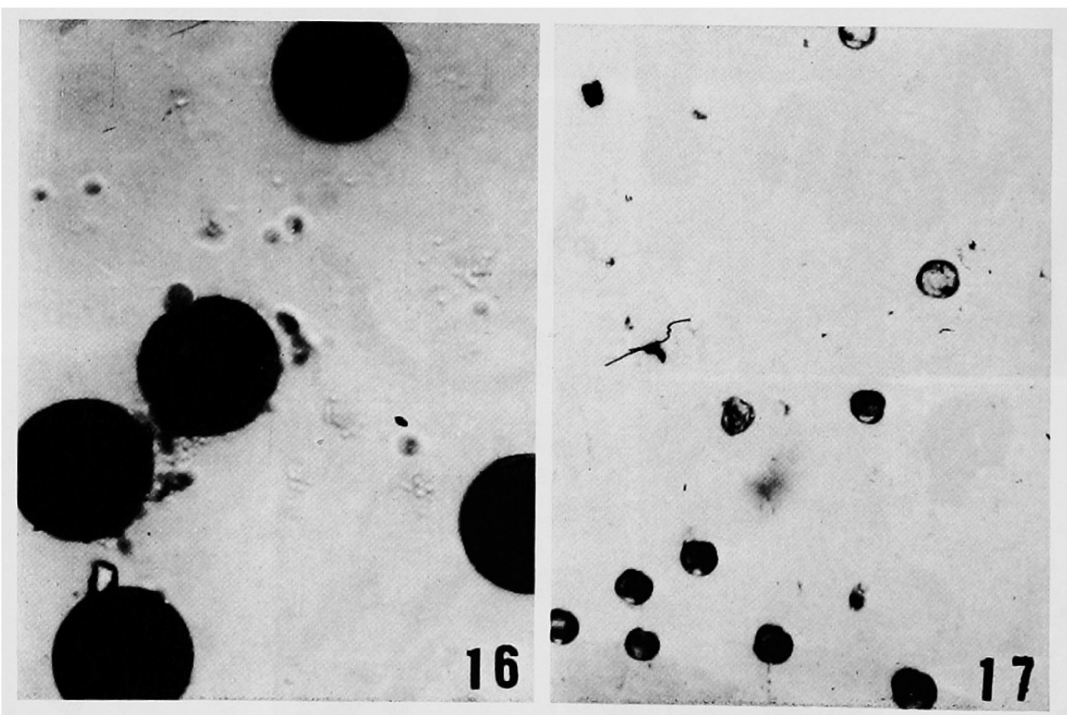

Figs. 16-17. Pollen grains of Nigella sativa. $\quad \times 480 . \quad 16$, normal stained pollen grains in control plants. 17 , stained and unstained small sized pollen grains in the phenotypically marked aberrant plant.

Apart from variable numbers, ring and chain configurations of bivalents were also noted in the PMCs of the aberrant plant (Figs. 7, 10 and 11). Occurrence of a ring-like quadrivalent (Fig. 14) in few PMCs (5.3\%) suggests possible interchanges involving four chromosomes. 
Pollen sterility was remarkably higher $(78.5 \%)$, while size of both stained and unstained pollen was considerably smaller $(10.05 \mu \pm 0.2)$ in the aberrant plant than the respective values of the control plants $(2.2 \%-3.5 \%, 39.8 \mu \pm 0.6)$. Moreover, functional inability of the stained (fertile) pollen has been evidenced by the formation of only rudimentary seeds in the marker plant. Crossing experiments performed with pollen from the control plants yielded only abortive seeds, which gives an indication of defective nature of female gametophytic tissues. As compared to controls (Fig. 16), pollen sterility and extreme reduction in pollen size (Fig. 17) observed in the marked plant may be the outcome of disturbances in pollen development, as a possible consequence of meiotic instability or due to some other unknown factor(s). In this context, however, studies of anaphasic behaviour of meiotic chromosomes could have thrown additional light.

The present phenotypically aberrant plant and a trisomic plant were recovered from the segregating selfed progeny of a EMS-induced lax branching mutant $\left(\mathrm{M}_{2}\right)$, showing cytomixis and aneuploid variations. Trisomic condition has already been reported (Datta and Biwsas 1984) as a consequence of cytomixis and the same phenomenon can be ascribed for the origin of the present aberrant plant. Possibly, marked phenotypic variations in the aberrant plant are the manifestation of some cytological adversities resulted due to cytomixis and the meiotic instability leading to chromosomal mosaicism in the microsporocytes of the plant has arisen due to premeiotic mitotic disturbances. Meiotic chromosomal variations due to premeiotic mitotic abnormalities have also been reported in other plant species (Sachs 1952, Venkateswarlu and Raja Rao 1979, Narain 1980).

\section{Summary}

A phenotypically aberrant and sterile plant was identified in the selfed progeny of a EMS- induced $\mathrm{M}_{2}$ mutant (lax branching) of Nigella sativa L. (black cummin), showing cytomixis and aneuploid variations. The aberrant plant revealed aneuploid and polyploid variations in the meiotic cells with remarkably high sterility of pollen and extreme reduction of pollen size. Such meiotic variations occurring within the microspore mother cell of the same microsporophyll have been referred to as "chromosomal mosaic". It is most likely that the aberrant plant has arisen due to some cytological anomalies as a consequence of cytomixis, and the meiotic instability manifested by the plant leading to chromosomal mosaic might have occurred due to premeiotic mitotic disturbances.

\section{Acknowledgement}

The authors are highly indebted to Dr. N. K. Bhattacharyya (K.U.) and Prof. S. Sen (B.C.K.V.) for their valuable suggestions and also to University of Kalyani for financial assistance to the senior author.

\section{References}

Datta, A. K. and Biswas, A. K. 1983. X-ray sensitivity in Nigella sativa L. Cytologia 48: 293- 
303.

— and - 1984. Cytomixis and a trisomic in Nigella sativa L. Cytologia 49:437-445.

Fukumoto, M. 1962. Nuclear instability and chromosomal mosaicism in higher-ploids of Solanum species and hybrids. Jap. J. Bot. 18: 19-53.

Guildenhuys, P. and Brix, K. 1958. Cytological abnormalities in Pennisetum dubium. Heredity 12: $441-452$.

Narain, P. 1980. Chromosomal mosaicism in the microsporocytes of Gloriosa. Cytologia 45: 271-279.

Nielsen, E. L. 1961. Cytology and fertility of Agroehymus turneri. Cytologia 26: 137-154.

Sachs, L. 1952. Chromosome mosaics in experimental amphidiploids in the Triticineae. Heredity 6: $157-169$.

Thompson, M. M. 1962. Cytogenetics of Rubus III. Meiotic instability in some higher polyploids. Amer. J. Bot. 49: 575-582.

Venkateswarlu, J. and Raja Rao, K. G. 1979. Chromosome numerical mosaicism in a tetraploid interracial hybrid of Physalis angulata L. J. Cytol. Genet. 14: 5-7. 